\title{
El mundo rural a través del léxico de José Antonio Muñoz Rojas
}

PILAR FERNÁNDEZ MARTÍNEZ

UNIVERSIDAD CEU SAN PABLO (MADRID)

ABSTRACT: This paper examines some of the rural terminology used by Antonio Muñoz Rojas in Las cosas del campo. These are words that refer to an almost disappeared reality and that we want to document Approximately fifty terms with the particular feature of not having been defined by an academic dictionary are analyzed, either because they have not had a proper entry or because the specific meaning of the term is not acknowledged. The words that, though registered by the DRAE (Diccionario de la Real Academia Española), carry a specific meaning in the land of the poet are also included. Each vocabulary entry comprises the grammatical category, its intended, and a brief extract from the poetic prose of the writer for its contextualization.

Keywords: rural lexicon, poetic prose, Muñoz Rojas.

RESUMEN: En el presente trabajo se analiza una parte de la terminología rural utilizada por José Antonio Muñoz Rojas en Las cosas del campo. Son voces que aluden a una realidad ya casi desaparecida y de la que queremos dejar constancia. Estudiaremos cerca de medio centenar de términos que tienen la particularidad de no haber sido definidos en un diccionario académico, bien sea por no haber tenido entrada o bien porque el significado concreto del término no está contemplado en él. También incluiremos aquellas voces que, aunque recogidas por el DRAE (Diccionario de la Real Academia Española), presentan un valor concreto en la tierra del poeta. En cada lema se registrará la categoría gramatical, su acepción o acepciones, así como un breve fragmento en el que se inserta dicha voz y que constituye una pequeña muestra de la prosa poética de José Antonio Muñoz Rojas.

Palabras clave: léxico rural, prosa poética, Muñoz Rojas. 


\section{Introducción}

Tan solo tenía 37 años cuando el poeta antequerano José Antonio Muñoz Rojas (1909-2009) creaba Las cosas del campo: un diario escrito a pluma en el que, desde el 20 de marzo de 1946 hasta el 21 de mayo de 1947, recorre y hace suya esa tierra que le acompaña cada día desde su lugar de residencia, «La Casería del Conde».

Como él mismo dice, en la edición de 1999, la obra responde a «la necesidad de rellenar unas hojas en blanco de papel del siglo XVIII de un libro encuadernado en piel que me había regalado mi hermano mayor Juan a este fin» (Hernández Mirón, 2011: 221). No hay, pues, en la gestación de esas páginas, el deseo último de que saliesen más allá del propio entorno familiar. De hecho, el manuscrito quedó inédito hasta que en 1950 sus amigos malagueños Pepe Salas, Bernabé Fernández Canivell y Alfonso Canales le pidieron que escribiera para una colección poética que dirigían. Surgió así la primera edición de la obra, la cual constaba de un número muy limitado de ejemplares, un total de 200; esto hizo exclamar al maestro Dámaso Alonso:

¿Va a quedarse en 200 ejemplares para 200 exquisitos, privilegiadísimos lectores? Sería un egoísmo pecaminoso. No; este libro tiene que volar en muchísimos ejemplares. ¡Que vuele por toda España, que los españoles no se ilusionen con trampantojos ni artificios, que aprendan la belleza de lo elemental y de lo primario! Sí; yo quisiera que Las cosas del campo, en edición copiosa, llevaran a muchos corazones la serenidad y el consuelo que han traído al mío. (Dámaso Alonso, 1975: 303-304)

Los deseos del académico no tardaron en hacerse realidad, ya que, dos años más tarde, en 1953, salió una segunda edición aumentada, que contó con un total de mil ejemplares. Sin embargo, tendrían que pasar veintitrés años para que, en 1976, la editorial Destino, por sugerencia de Elena Quiroga, volviera a editar Las cosas del campo. En esta edición, el poeta introducía una advertencia-«Advertencia en 1975»- en la que nos hablaba con nostalgia de un campo que empezaba a desaparecer y de unas voces que pasaban, ya entonces, a ser desconocidas: «Ni Pensador, ni Velador, ni sus oficios, existen ya [...] No quedan ni bielgos, ni barcina, ni ninguno de aquellos instrumentos de verano que hacían vivas las eras. Apenas si sus nombres se conocen» (Muñoz Rojas, 2007: 11).

Esta «Advertencia» se mantiene en la $4^{\mathrm{a}}$ y última edición realizada por Pre-Textos en $1999,{ }^{1}$ y es la que motiva las páginas que escribimos a continua-

1. Esta edición cuenta con siete reimpresiones. La última corresponde al año 2009. 
ción. Queremos centrarnos en el análisis de esos términos que «apenas si [...] se conocen» y rescatarlos de un olvido injusto. Muchos de ellos nunca pasaron las fronteras de un diccionario académico y, si lo hicieron, no siempre fueron recogidos con el valor diatópico y preciso del término.

Para ello hemos consultado distintos trabajos teóricos y lexicográficos (vid. bibliografía) y, cuando estos no han sido suficientes, hemos acudido al trabajo de campo, a la encuesta, a la consulta directa a quienes tuvieron la dicha de compartir el entorno y la amistad del poeta. Deseo destacar, en este sentido, las indicaciones de Antonio Carvajal, poeta y amigo personal de José Antonio Muñoz Rojas, al ayudarme a entender el significado de algunas voces como avinatar o mandar y barajar olivos. Ellos conocieron, como nadie, el valor y la magia que la palabra adquiría en boca del antequerano:

Mi padre, agricultor y amante de la lectura, encontraba allí conformado, con palabra precisa y mirada bondadosa, un mundo que él sentía y no podía expresar. Supe, entonces, que es altísima misión de la poesía prestar palabra necesaria a quienes no saben pronunciarla por sí mismos. (Antonio Carvajal, 2000: 6)

\section{Análisis lexicográfico}

«Creo que este tirón del campo labrador, no solo de la naturaleza paisaje, ha sido con lo religioso, un fuerte conformador de mi vida» decía Muñoz Rojas en La gran musaraña (1994:115). El poeta conoce la naturaleza que le rodea, sabe que las plantas tienen un nombre preciso, que la mata del anís en su tierra es la matalahúga, y que no existe nada comparable con la belleza de los nerdos, de la lechitrezna o de los Zapaticos del Niño Dios... Sabe -porque conoce a Machado- que no hay nada más estúpido que silenciar el nombre directo de las cosas, y sabe que esos nombres quedarán como un testimonio frente al paso del tiempo. Solo adentrándonos en ellos, el hombre de hoy, tantas veces desarraigado de su propia tierra, podrá lograr vislumbrar la existencia de una realidad que jamás debería caer en el olvido: «¡Ay de los que lo olvidaren!» (Muñoz Rojas, 2007:11).

\section{1. «Palabras viejas y sonoras: un léxico prácticamente perdido en algún} rincón de la lengua» (Marín Cejudo, 2009):

Con el fin de ofrecer una visión de conjunto, presentaremos el léxico estudiado agrupándolo en cinco campos semánticos: Tierras, plantas y flores del campo; aves; labores del campo; hombres del campo; aperos 
y construcciones. De este modo, las palabras se convierten en muestra del gran acervo léxico que el escritor antequerano ofrece en su obra:

TIERRAS, PLANTAS Y FLORES DEL CAMPO

Albero: (s.m.) Terreno de secano, y especialmente tierra blanquecina en altos y lomas (DRAE, vid. albar). En Andalucía recibe este nombre el terreno calizo, la arcilla gredosa: «Los alberos ven olivos fruteros, siempre frescos y enramados» (Muñoz Rojas, 2007: 79).

Albina: (s.f.) En Andalucía, tierra albariza, ${ }^{2}$ aunque también se llama así a la hierba que al secarse queda de color blanco: «Tierras duras, alberos y polvillares, breves bugeos, largos cubriales; aquí se riza una loma, allá se quiebra una cañada, se extiende una albina, tiembla un sisón de vuelo lento» (Muñoz Rojas, 2007: 13).

Bugeo/Bujeo: ${ }^{3}$ (s.m.) El bujeo, o tierras negras, es un término agronómico utilizado para denominar los típicos suelos de cultivo de secano de la campiña andaluza. El color de estos suelos va desde el pardo amarillento al pardo gris oliva y de un gris oscuro a prácticamente negro, en función de su composición. El bujeo es un suelo pesado, difícil de trabajar. En Málaga se llama así al «terreno presto a endurecerse con frecuencia»: «Tierras duras, alberos y polvillares, breves bugeos» (Muñoz Rojas, 2007: 13). «Surco a surco, el braván va borrando el amarillo del campo, vistiéndolo de colores severos, blanquecino en los alberos, rojizo en los polvillares, grisáceo en los riquísimos bujeos» (Muñoz Rojas, 2007: 94).

Cubrial: (s.m.) Tierra cobriza o rica en cobre. Clara Martínez Mesa considera este término como un neologismo creado por el poeta (Muñoz Rojas, 2008: 400): «Tierras duras, alberos y polvillares, breves bugeos, largos cubriales; aquí se riza una loma, allá se quiebra una cañada, se extiende una albina, tiembla un sisón de vuelo lento» (Muñoz Rojas, 2007: 13).

2. El DRAE nos ofrece un significado distinto: «Estero o laguna que se forma con las aguas del mar en las tierras bajas que están inmediatas a él. 2. f. Sal que queda en estas lagunas».

3. Como en otras ocasiones, Muñoz Rojas parece jugar con la ortografía de las palabras $-g / j ; r / l$; $h / \varnothing$-, pues el término bugeo no lo encuentro recogido en ninguna de las obras consultadas. Aunque bujeo sí tiene entrada en el DRAE, no se recoge la acepción utilizada por Muñoz Rojas, referida a un tipo de suelo de cultivo propio de Andalucía. Alcalá Venceslada (1951) remite al término bufeo, «Tierra de labor muy arcillosa que al resecarse en verano forma grandes grietas». Sí hemos hallado el término bujeo como un andalucismo en los diccionarios académicos de 1970 a 1992 (NTLLE-Nuevo Tesoro Lexicográfico de la Lengua Española). En todos ellos se remite al término buhedo, término que aparece ya en la edición de 1770 con el significado de «tierra gredosa», significado que permanece hasta la edición de 1914. 
Cudrial: (s.m.) Se dice de la tierra que una vez seca, es muy dura y compacta, ofreciendo gran dificultad para toda clase de labores: «Los alberos ven olivos fruteros, siempre frescos y enramados, los cudriales los desmedran, los polvillares los asolan» (Muñoz Rojas, 2007: 79).

Grama: (s.f.) Planta medicinal de la familia de las gramíneas, con el tallo cilíndrico y rastrero, que echa raicillas por los nudos. Tiene hojas cortas, planas y agudas, y flores en espigas filiformes que salen en número de tres o de cinco en la extremidad de las cañitas de dos decímetros de largo (DRAE).

La grama se cría al borde de los caminos y en tierras de labranza. Una vez que crece entre el cultivo es difícil eliminarla, pues o se quita bien la raíz o vuelve a crecer.

Es conocida también con los nombres de «gramilla blanca», «gramilla dulce», «trigo rastrero», «pata de perdiz» $\mathrm{y}$ «pata de gallina»: «Van desmenuzando terrones, arrancando la grama tenaz, alisando las calles entre las matas» (Muñoz Rojas, 2007: 63-64).

Jaramagal: (s.m.) Sitio poblado de jaramagos: ${ }^{4}$ «El campo es una gran sinfonía en amarillo donde apenas dan una leve nota, blancos de nievecillas, morados de lenguazas y nazarenos, rojos de amapolas y aquellas florecillas, que el jaramagal, que llega ya a la cruz de los olivos y a los ijares de los caballos, permite florecer» (Muñoz Rojas, 2007: 47).

Lechín: (s./adj.m.) Se dice de una variedad de olivo (DRAE). Este puede presentar a su vez formas distintas, tales como el lechín ecijano, de hoja fina larga, color verde tostado y fruto fino y largo, y el lechín jaenero, de hoja oscura y fruto menudo y redondo (Alcalá Venceslada, 1951; Alvar Ezquerra, 2000): «Todavía en medio de los ordenados olivares de hoy, sobresalen a veces restos de olivos viejos de casta distinta, lechines, manzanillos, injertos algunos en acebuches por las cercanías de montes y cañadas» (Muñoz Rojas, 2007: 79-80).

Lechitrezna: (s.f.) Hierba de flores color verde muy brillante. Es conocida también con nombres como chiriguela, hierba de la purga, piñoncillos, recheluera, etc. Se caracteriza fundamentalmente por que todas las partes de la planta contienen un látex blanco y muy viscoso, parecido a la leche y de ahí toma su nombre común. Su nombre científico es Euphorbia serrata. Es muy conocida en cultivos, en especial de vid, donde es considerada mala hierba.

Alvar Ezquerra (2000) recoge, en Andalucía, las variantes lechiruela, «Planta silvestre cuya savia es blanca» y lechiterna «Variedad de planta que se caracteriza por ser muy nociva o venenosa»: «Qué inesperadas [...] las yerbas

4. El DRAE define jaramago, pero no así jaramagal, voz que no tiene entrada en el diccionario académico. 
ignoradas [...], la que dicen lechitrezna, [...] los jaramagos, y las mil plantas que llaman yerbas del campo» (Muñoz Rojas, 2007: 25).

Lenguaza: (s.f.) Planta anual de la familia de las borragináceas, muy vellosa, con tallo erguido, de seis a ocho decímetros de altura, hojas lanceoladas, enteras, las inferiores con pecíolo, sentadas las superiores, y todas erizadas de pelos rígidos, flores en panojas de corola azul y forma de embudo, y fruto seco con cuatro semillas rugosas. Abunda en los sembrados, y sus flores forman parte de las cordiales. El DRAE la recoge bajo el lema lengua de buey: « $\mathrm{O} \mathrm{Oh}$, jaramagos, lenguazas, zapaticos, nazarenos, ignoradas yerbas del campo!» (Muñoz Rojas, 2007: 26).

Manzanillo (olivo): (s./adj. m.) Olivo que da aceituna manzanilla (DRAE). Alcalá Venceslada (1951) remite a manzanillo de agua y lo define como una casta de olivo de hoja ancha oscura y fruto redondo, muy propio para el adobo: «Todavía en medio de los ordenados olivares de hoy, sobresalen a veces restos de olivos viejos de casta distinta, lechines, manzanillos, injertos algunos en acebuches por las cercanías de montes y cañadas» (Muñoz Rojas, 2007: 79-80).

Maojo: (s.m.) 1. En Málaga, farfolla, flor del maíz (vid. ALEA-Atlas Lingüístico y Etnográfico de Andalucía- I, 105: Ma 500). 2. Cascarilla del trigo y demás cereales (Granada): «Los mulos lo conocen y entregan sus cabezas, se alinean y José monta a mujeriegas en el más viejo. Saben que los llevará donde abunde la espiga olvidada, donde el maojo esté más tierno» (Muñoz Rojas, 2007: 62).

Matalahúga: (s.f.) Planta anual de la familia de las umbelíferas, que crece hasta unos $30 \mathrm{~cm}$ de altura, con tallo ramoso, hojas primeramente casi redondas y después hendidas en lacinias, flores pequeñas y blancas. Tiene por frutos semillas aovadas, verdosas, menudas y aromáticas. El DRAE remite al lema matalahúva y de aquí a anis: «La matalahúga la siembra la luna. Por marzo, cuando hace todavía frío y nadie la ve, la luna se aprovecha y la siembra. Si no, ¿de dónde la plata que es pura plata lunar de la flor, por junio?» (Muñoz Rojas, 2007: 73).

Nazareno: (s.m.) Planta herbácea perenne de la familia de las liliáceas, con las hojas basales, lineares, y flores violetas que se reúnen en racimos. Crece de manera espontánea. Su nombre científico es Muscari comosum: «Los que llaman nazarenos, la que dicen lechitrezna, $[\ldots]$ los jaramagos, $[\ldots] ; O \mathrm{Oh}$ nobles yerbecillas!» (Muñoz Rojas, 2007: 25).

5. El DRAE define este tipo de aceituna como una especie «pequeña, muy fina, que se consume en verde, endulzada o aliñada». 
Nerdo: (s.m.) Planta de las umbelíferas, anual, que puede alcanzar los $100 \mathrm{~cm}$. Las hojas están muy divididas en segmentos filiformes y podrían presentar confusión con las del hinojo. Se diferencia de este, a pesar de la falta de olor tan característico, porque el pedúnculo de las umbelas no es tan largo y toda la planta en flor tiene forma redondeada. Las umbelas son compuestas, sin brácteas y de flores amarillas. Los frutos tienen el contorno con forma de huevo y están comprimidos lateralmente. Se da en la zona meridional y la época de floración va de mayo a agosto. Su hábitat son los campos baldíos. Su nombre común es neldo. ${ }^{6} \mathrm{Su}$ nombre científico es Ridolfia segetum.

Alcalá Venceslada (1951) y Alvar Ezquerra (2000) recogen el lema nerdo, y lo definen como «eneldo, planta espontánea muy común en las marismas del Guadalquivir»: «Entre el verde metálico de los maizales, entre el azulenco de los garbanzos, entre el amarillo de los nerdos, el finísimo blanco de la matalahúga» (Muñoz Rojas, 2007: 73).

Ojiblancar: (s.m.) Terreno sembrado de ojiblancos: ${ }^{7}$ "Ya nadie se acuerda de quién puso el Ojiblancar, ni de qué manos abrieron sus hoyos, ni cuáles talaron sus primeros ramones» (Muñoz Rojas, 2007: 106).

Ojiblanco (olivo): (s.m.) Olivo de hoja muy verde fina y blanca. Su área de influencia se extiende por Andalucía, en concreto por el este de la provincia de Sevilla, sur de Córdoba y todo el norte de la provincia de Málaga. Puede suponer el $16 \%$ del olivar andaluz. También se le conoce en Andalucía bajo el nombre de lucentino, por los reflejos metalizados que tienen sus hojas cuando les da el sol. Las aceitunas hojiblancas sirven tanto para aceitunas de mesa negras por la firme textura de su pulpa, como para la producción de aceite: ${ }^{8}$ «Estas diligentes filas de ojiblancos que no se acaban y a quienes no detienen más que las peñas en las herrizas y los limos de los ríos donde llegan a correr» (Muñoz Rojas, 2007: 80).

Pegujal: (s.m.) En Málaga recibe este nombre el trozo de terreno generalmente plantado de trigo: ${ }^{9}$ «El calor es tremendo [...] Los pegujales que quedan en pie, humillan la espiga y piden la hoz para el descanso sobre la tierra que ya

6. La transformación de neldo a nerdo se explica fácilmente por la confusión de las implosivas $/ \mathrm{r} /$ y / $/$ al final de sílaba o de palabra, fenómeno frecuente en el andaluz.

7. Se trata de un neologismo formado sobre la base del sustantivo ojiblanco más el sufijo -ar («lugar donde existe o abunda») (Cfr. con otros sustantivos como habar, melonar, olivar, etc.)

8. En las obras consultadas alternan las grafías ojiblanco y hojiblanco (vid. Alcalá Venceslada (1951), Bustos (1986) y Alvar Ezquerra (2000)

9. Este valor es recogido por Juan Cepas en su Vocabulario popular malagueño (2005), frente al significado general que ofrece el DRAE. 
no les puede dar nada. Vienen los barcinadores y van cargando las gavillas en el carro» (Muñoz Rojas, 2007: 57).

Polvillar: (s.m.) Tierra arenosa. En Málaga se llama también así a la tierra de mala calidad. Alvar Ezquerra (2000) recoge también otros valores propios de las tierras andaluzas: en la Sierra de Cazorla se llama así a «la tierra de fondo, fértil y fácil de labrar» y en Córdoba, (usado siempre en plural) «tierras rojizas con muchas piedrecitas»: «Tierras duras, alberos y polvillares, [...]; aquí se riza una loma, allá se quiebra una cañada» (Muñoz Rojas, 2007:13).

Ramón: (s.m.) Ramaje que resulta de la poda de los olivos y otros árboles (DRAE). En Jaén recibe este nombre «la parte del olivo con hojas»; en Málaga y Córdoba, «el ramojo del olivo»: «El rumor pasa y tras él quedan enhiestos los ramones, quieto el aire» (Muñoz Rojas, 2007: 103).

Realenga: En Córdoba, Granada y Málaga, camino para el ganado trashumante (vid. ALEA, II, 445): «Yo me estremezco andando estas realengas, cruzando estas lindes, asomándome a estas herrizas» (Muñoz Rojas, 2007: 14).

Tempero: (s.m.) Sazón y buena disposición en que se halla la tierra para las sementeras y labores (DRAE). En Andalucía se llama también así al estado de la atmósfera, temperatura, humedad y sensación que producen ambas: «Todo depende, como dicen aquí, del tempero» (Muñoz Rojas, 2007: 81).

Trama: (s.f.) Florecimiento y flor de los árboles, especialmente del olivo (DRAE). También se llama así al «botón floral del olivo» y a «la primera fase de formación del fruto del olivo, después de caerse la flor»: «Pasó el reinado del jaramago. Pasó la trama en los olivos. Reinan los nerdos; el sembrado es rastrojo» (Muñoz Rojas, 2007: 55).

Zapatico (zapatico del Niño Dios): (s.m.) Planta herbácea anual, de tallo bajo, frágil y ramificado, con hojas divididas de color blanco. Presenta flores tubulares unidas en largos racimos violáceos. Es también conocida con los nombres de camisita de la virgen, camisitas del Niño Jesús, pañalitos del Niño Jesús, penitentes, piececitos, zapaticos de la Virgen (así la recogen Alvar Ezquerra y Alcalá Venceslada), zapaticos de Nuestro Señor, zapaticos de San José...

Clara Martínez Mesa (Muñoz Rojas, 2008: 405-406) afirma que se trata de un nombre utilizado popularmente en Andalucía para designar flores menudas, generalmente de color blanco: «¿Hasta cuándo voy a ignorar vuestros nombres? [...] Los que llaman nazarenos, la que dicen lechitrezna, los zapaticos del Niño Dios (que son el prodigio de finura con que Dios pisa la tierra), los jaramagos, y las mil plantas que llaman yerbas del campo» (Muñoz Rojas, 2007: 25). 
Aves:

Pájara: (s.f.) Hembra de la perdiz. El DRAE lo recoge como un andalucismo propio, fundamentalmente, de la provincia de Jaén: «Por mayo se caza la pájara. Hay que aprovechar el celo del macho, diez días escasos, recién desemparejado y en estado de merecer» (Muñoz Rojas, 2007: 77).

Primilla: (s.f.) El DRAE define esta voz como un andalucismo con el que se nombra al cernícalo. En Málaga recibe también este nombre el ave de rapiña ligeramente mayor que el cernícalo, pero de características y costumbres casi idénticas (ALEA, II, 422n: Ma408): «Enero es así [...] ni apenas pájaros. Alguna avefría silenciosa, alguna primilla a lo suyo, dos lentos grajos. Todo se está quieto» (Muñoz Rojas, 2007: 111).

\section{LABORES DEL CAMPO}

Aceitunería: (s.f.) Recogida de la aceituna: ${ }^{10}$ «Pocas veces hará la tierra más suyos a los hombres que en las aceitunerías» (Muñoz Rojas, 2007: 101).

Avinatar: (v.) Tomar gusto ácido las frutas excesivamente maduras: ${ }^{11}$ «La aceituna sin madurar se avinata y empequeñece» (Muñoz Rojas, 2007: 110).

Barajar (olivos): (v.) Poner orden, componer, arreglar los olivos: «¿Qué haces? - Lo de siempre mandando olivos. No hay quien los baraje» (Muñoz Rojas, 2007: 10).

Careo: (s.m.) Acción y efecto de carear: el hecho de dirigir el ganado hacia alguna parte y de apacentarlo: ${ }^{12}$ "Ahora se puede salir al campo, tumbarse en la era, encararse con las estrellas, escuchar el corazón del mundo. Ahora suena el agua en la reguera, la copla en la realenga, los pasos de las bestias en el careo» (Muñoz Rojas, 2007: 60).

Enlutar (las rejas): (v.) Dícese cuando el arado mete la reja en la tierra y esta va pegándose alrededor, cubriendo y oscureciendo el hierro, impidiendo que se puedan hacer bien los surcos y que el labrador pueda arar la tierra: ${ }^{13}$

10. Fernández-Sevilla alude, precisamente, a la falta de lexema para referirse a este hecho: «El dialecto no posee una forma léxica simple y específica para el semema "recoger aceitunas", inexistente, por otra parte, en la lengua oficial y en las demás lenguas románicas» (Fernández-Sevilla, 1975: 265).

11. Alvar Ezquerra (2000) recoge con este valor la voz avinarse.

12. El DRAE recoge este valor (vid. acepciones 2 y 3 de carear), sin embargo no lo considera un andalucismo. No obstante, los léxicos andaluces consultados lo incluyen como un término propio de aquella zona (Alcalá Venceslada, 1951).

13. Se trata de un neologismo del que se hace eco el autor y que utilizará en diversas ocasiones: «Después de las lluvias de invierno, las tierras no demasiado pegadizas suelen estar buenas y no enlutan la reja que entra bien y con fruto» (Muñoz Rojas, 2007: 103). 
«Vamos a caballo y oigo tras de mí:-Como está la tierra tan pegajosa se enlutan las rejas y no se puede arar. "Se enlutan las puntas de las rejas, se enlutan las puntas de las rejas", me quedo yo pensando maravillado de la justeza de la expresión en estos labios» (Muñoz Rojas, 2007: 38).

Mandar (olivos): (v.) Componer, arreglar y poner orden en los olivos: ${ }^{14}$ "¿Qué haces? - Lo de siempre mandando olivos. No hay quien los baraje» (Muñoz Rojas, 2007: 10).

\section{HOMBRES DEL CAMPO}

Aventador: (adj./s. m.) La persona encargada de separar la tierra, las hojas de la aceituna avareada en la llamada criba: ${ }^{15}$ "Voces, alguna copla, el ruido de un banco que se cierra, el manoteo rápido sobre las hojas, el aleteo del aventador, la caída continua y mullida de la aceituna, como una cascada negra, en los sacos») (Muñoz Rojas, 2007: 101).

Barcinador: (s.m.) Dícese del hombre que barcina (DRAE). Es un andalucismo utilizado para nombrar al hombre que se encarga de coger las gavillas de la mies, echarlas en el carro y conducirlas a la era: «Los pegujales que quedan en pie, humillan la espiga y piden la hoz para el descanso sobre la tierra que ya no les puede dar nada. Vienen los barcinadores y van cargando las gavillas en el carro» (Muñoz Rojas, 2007: 57).

Cagarrache: (s.m.) Operario de la almazara dependiente del maestro o contramaestre (DRAE): ${ }^{16}$ «Eran aquellos olivos de molino de viga, con largos husillos de ciprés o nogal, manejados por poco más que maestro y cagarrache que duraban lo que Dios quería, porque no eran tiempos de prisa, como acomoda a los olivos que maldito el caso que hacen del tiempo» (Muñoz Rojas, 2007: 80).

Maestro: (s.m.) Trabajador de la almazara que dirige la fábrica: «Eran aquellos olivos de molino de viga, con largos husillos de ciprés o nogal, manejados por poco más que maestro y cagarrache» (Muñoz Rojas, 2007: 80).

Pensador: (s.m.) En los cortijos de Andalucía, mozo encargado de dar los piensos al ganado de labor (DRAE, vid. pensador 2): «Ni Pensador, ni Velador, ni sus oficios, existen ya. Los mulos se acabaron y las cuadras están desiertas y sin rumores de piensos» (Muñoz Rojas, 2007: 10).

14. El poeta Antonio Carvajal, amigo personal de Muñoz Rojas, plantea que esta expresión puede ser considerada sinónima de barajar olivos.

15. El DRAE ofrece el significado general «Dicho de una persona: Que avienta y limpia los granos».

16. Almazara: 1. f. Molino de aceite (DRAE). 
Velador: (s.m.) El pensador, el cuadrero, en los aperos de mulas o bueyes, el gañán que da el pienso por las noches: «Del pensador al velador va lo que va del invierno al verano, de largas noches en la cuadra a largas noches al raso, bajo las estrellas» (Muñoz Rojas, 2007: 61).

\section{APEROS Y CONSTRUCCIONES}

Barcina: (s.f.) 1 Herpil: saco de red de tomiza, con mallas anchas, destinado a portear paja, melones, etc. 2. Carga o haz grande de paja (DRAE). Se trata de una voz típicamente andaluza, de origen desconocido pero muy antiguo, a juzgar por la fortuna que la palabra tuvo ya en mozárabe y en árabe magrebí (FernándezSevilla, 1975): «No quedan ni bielgos, ni barcina, ni ninguno de aquellos instrumentos de verano que hacían vivas las eras» (Muñoz Rojas, 2007: 11).

Bielgo/Biergo: (s.m.) Instrumento para aventar, compuesto de un palo largo, de otro de unos $30 \mathrm{~cm}$ de longitud, atravesado en uno de los extremos de aquel, y de cuatro o más fijos en el transversal, en forma de dientes.

El DRAE remite a la voz bieldo. Los lemas bielgo/biergo son comunes en Andalucía. La variante biergo $^{17}$ (marcada en cursiva en la obra de Muñoz Rojas) responde a la confusión de las consonantes implosivas /r/ y /1/ -rasgo característico del andaluz-, y es la que recoge Alcalá Venceslada (1951) en su Vocabulario Andaluz. Fernández-Sevilla señala las variantes fonéticas bielgo y biergo como las más abundantes del término bieldo. Biergo presenta una proporción con la forma etimológica bielgo de 126/7 (Fernández-Sevilla, 1975: 213): «Crujen los trillos, salta la gavilla, dormitan los gañanes. Al primer anuncio de brisa ya están aventando. El biergo y el viento hacen cada uno lo suyo y el grano cae» (Muñoz Rojas, 2007: 56).

Braván: (s.m.) 1. Antiguo arado de hierro con dos ruedas, para tiro animal. 2. Arado metálico provisto de doble sistema de piezas activas que pueden voltear la tierra tanto a la derecha como a la izquierda.

Muñoz Rojas usa siempre este término escrito con $v$, frente a la variante con $b$ recogida por Alcalá Venceslada (1951) y por Alvar Ezquerra (2000): «una clase de arado que hace una labor muy honda». La Academia no recoge ninguna de las dos variantes gráficas. No obstante, a pesar de que la variante con $v$ no la he hallado en los léxicos andaluces consultados, es fácil encontrarla en páginas y folletos referidos a tipos de arados: «Va siendo la hora del braván, de que los membrillos colmen el aire con su aroma y de que caiga el primer fruto de los nogales» (Muñoz Rojas, 2007: 88).

17. El DRAE considera esta voz propia de Extremadura. 
Cauchín: (s.m.) Canal por donde va el agua de los molinos: «Pero [...] puntualmente vendrán [los olivos] con su aceituna el año que les toque y generosamente correrá el aceite por cauchines en los molinos...» (Muñoz Rojas, 2007: 79).

Choza: (s.f.) Cabaña (DRAE). Fernández-Sevilla siente esta voz como una variante de chozo (junto a otras como chociya, chocita, chozón...) y la define como 'cabaña formada de estacas y cubierta de ramas o paja, en la cual se recogen los pastores y gente del campo' (Fernández-Sevilla, 1975: 186). Alvar Ezquerra (2000) señala que en Málaga llaman así a la 'cabaña para guardar melones o maíz' (ALEA II, 450n: Ma 101): «En la chocilla los niños del melonero esperan impacientes el primer fruto, como un regalo de la tierra» (Muñoz Rojas, 2007: 63).

Dornillo: (s.m.): Mortero grande de madera, normalmente de olivo, para majar los alimentos y los condimentos, con mazo de madera y en el que tradicionalmente se sirve la pipirrana y se hace el salmorejo y el gazpacho. ${ }^{18}$ Alvar Ezquerra (2000) señala que en Málaga se llama así a la «escudilla»: «Pero [...] puntualmente vendrán [los olivos] con su aceituna el año que les toque y generosamente correrá el aceite por cauchines en los molinos y blandamente se derramará en dornillos y rebanadas» (Muñoz Rojas, 2007: 79).

Horca: (s.f.) Palo que remata en dos o más púas hechas del mismo palo o sobrepuestas de hierro, con el cual los labradores hacinan las mieses, las echan en el carro, levantan la paja y revuelven la parva (DRAE).

Julio Fernández-Sevilla (1975) señala que la diferencia fundamental entre la horca y el bieldo estriba en que el bieldo es totalmente de madera, mientras que la horca tiene los dientes de hierro. Además el bieldo es instrumento típico y casi específico de las funciones de la era, mientras que la horca se utiliza en otras muchas labores campesinas. En la parte oriental de Andalucía domina el término horca: «iQué bellos, estos instrumentos del verano! La horcas, las palas, los biergos, las carretas con sus varales» (Muñoz Rojas, 2007: 59).

Martaguilla: (s.f.) El DRAE define este término como un andalucismo con el que se nombra la jáquima de cuerda, ligera y fácil de quitar, que se usa especialmente para llevar de reata el caballo de silla: «Al volver los mulos de la besana ya está José esperándolos, las martaguillas y las trabas dispuestas, los cencerros preparados, la manta terciada: el relente de la madrugada la pide»» (Muñoz Rojas, 2007: 61-62).

Molino (de viga): (s.m.) Tipo de molino en el que la presión sobre la pasta de aceituna se ejecutaba por medio de un husillo que, firmemente unido a una

18. Aunque este término tiene entrada en el DRAE, no se recoge la acepción precisa con la que es usado en la obra de Muñoz Rojas. 
base de piedra, se hacía girar; el peso de la piedra hacía que esta no se levantase de su asiento, de tal forma que era la viga la que se desplazaba hacia abajo produciendo así presión sobre el cargo. El giro del husillo en sentido contrario permitía levantar la viga hasta su punto de reposo.

Sobre el origen y antigüedad del molino de viga no hay apenas documentación alguna pero la tradición atribuye su procedencia a la época musulmana: ${ }^{19}$ «Eran aquellos olivos de molino de viga, con largos husillos de ciprés o nogal, manejados por poco más que maestro y cagarrache que duraban lo que Dios quería» (Muñoz Rojas, 2007: 80).

Raspa: (s.f.) En Málaga, cuchilla recta con dos mangos utilizada para raspar y refilar: "iQué bellos, estos instrumentos del verano! La horcas, las palas, los biergos, las carretas con sus varales. Cuando llega la feria de mayo, se reponen. Vienen ya lisos, pero más lisos los pondrán espigas, raspas y manos» (Muñoz Rojas, 2007: 59).

\section{Conclusiones}

A lo largo de las páginas de Las cosas del campo hallamos un verdadero yacimiento de voces precisas, de términos certeros con los que Muñoz Rojas nos acerca a la belleza de un paisaje que se convierte en expresión de su propia alma. Voces que van cayendo en el olvido y en las que nosotros hemos querido detenernos. Del análisis de este casi medio centenar de palabras (48 voces estudiadas) podemos señalar que casi un $40 \%$ no han tenido entrada en el Diccionario académico, así: aceitunería, avinatar, barajar olivos, braván, bugeo, cauchín, cubrial, cudrial, enlutar las rejas, jaramagal, lechitrezna, mandar olivos, maojo, molino de viga, nerdo, ojiblancar, ojiblanco, polvillar y zapatico del Niño Dios.

Sí han sido recogidas por el DRAE, aunque no con la acepción con la que son utilizadas por el poeta, un $21 \%$ de ellas: albina, aventador, bujeo, dornillo, maestro, nazareno, pegujal, raspa, realenga, tempero, y velador.

El DRAE considera andalucismos los términos barcina, barcinador, martaguilla, pájara, pensador y primilla. El lema biergo es considerado por la DRAE propio de Extremadura, si bien, como hemos puesto de manifiesto, es una voz muy extendida en Andalucía.

El resto de las voces, aunque recogidas por el DRAE como términos del español estándar, hemos querido incluirlas, ya que en Andalucía presentan

19. Alvar Ezquerra (2000) define el molino de viga como «palo de 10 a 20 metros en la prensa de aceituna». 
unos valores propios que hemos tratado de reflejar: albero, bielgo, cagarrache, careo, choza, grama, horca, lechín, lenguaza, manzanillo (olivo) y matalahúga.

Muñoz Rojas nos deja en Las cosas del campo un verdadero tratado filológico, unido a toda la ternura de quien ama una tierra y tiene el don de saber revelarla. Cuando el poeta detiene su mirada en el campo y da nombre a las yerbas y a cuanto le rodea no solo describe lo que se presenta ante sus ojos, sino que lo hace suyo, lo incluye en su propia humanidad, y nos lo devuelve subjetivado en su mundo poético: «Eres un río de hermosura pasando, sonando, ajustándote a la noche, al día, a la estación. Por ti siento pasos antiguos, correr sangre de esta misma de mis venas. Todos somos como tú, algo que ni empieza ni acaba, como la hermosura o estos olivares cuyo fin nunca alcanzan mis ojos» («Las puertas del campo», Muñoz Rojas, 2007: 18).

\section{Referencias bibliográficas}

Alcalá Vencesladd, A. (1951): Vocabulario Andaluz, Madrid, Real Academia Española.

Alonso, D. (1975): Obras completas IV. Estudios y ensayos sobre literatura. Tercera parte. Ensayos sobre Literatura contemporánea, Madrid, Gredos.

Alvar Ezquerra, M. (2000): Tesoro Léxico de las Hablas Andaluzas, Madrid, Arco/Libros.

Alvar López, M.; A. Llorente; G. Salvador (1991): Atlas Lingüístico y Etnográfico de Andalucía (ALEA). 3 volúmenes, Madrid, Arco/Libros.

Bustos, E. (1986): La composición nominal en español, Salamanca, Ediciones Universidad de Salamanca.

Carvajal, A. (2000): «Ensayo introductorio» en Muñoz Rojas, J. A. (2000): Consolaciones del campo, Granada, Asociación de Padres Torres Bermejas. 5-7.

CEPas, J. (2005): Vocabulario popular malagueño, Málaga, Arguval.

Fernández-Sevilla, J. (1975): Formas y estructuras en el léxico agrícola andaluz: interpretación y estudio de 200 mapas lingüísticos, Madrid, CSIC.

Hernández Mirón, J. L. (2011): «La poética de José Antonio Muñoz Rojas» en Muñoz Rojas, J. A. (2011): Las cosas del campo, Madrid, Vitruvio.

Marín CeJudo, A. (2009): «Tierra y Comunión» en <http://www.elmundo. es> [09/10/2009]

Muñoz Rojas, J. A. (1994): La gran musaraña, Valencia, Pre-Textos.

- (2007): Las cosas del campo, Valencia, Pre-Textos. 
- (2008): Obra completa en verso, Estudio y edición de Clara Martínez Mesa, Valencia-Madrid, Pre-Textos.

Real ACademia Española (2001 a): Diccionario de la lengua española (DRAE), $22^{\mathrm{a}}$ ed., Madrid, Espasa-Calpe.

- (2001 b): Nuevo Tesoro Lexicográfico de la Lengua Española en $<\mathrm{http}: / /$ buscon.rae.es/ntlle/SrvltGUILoginNtlle> [9 de abril de 2012]. 